\title{
Preoperative Pain Level and Patient Expectation Predict Hospital Length of Stay After Total Hip Arthroplasty
}

\author{
Mohamad J. Halawi, MD a , Tyler J. Vovos, BS ${ }^{\text {b }}$, Cindy L. Green, PhD ${ }^{\mathrm{c}}$, Samuel S. Wellman, MD ${ }^{\mathrm{a}}$, \\ David E. Attarian, MD ${ }^{\mathrm{a}}$, Michael P. Bolognesi, MD ${ }^{\mathrm{a}}$ \\ a Department of Orthopaedic Surgery, Duke University Medical Center, Durham, North Carolina \\ b Duke University School of Medicine, Durham, North Carolina \\ c Department of Biostatistics and Bioinformatics, Duke University Medical Center, Durham, North Carolina
}

\section{A R T I C L E I N F O}

\section{Article history:}

Received 31 August 2014

Accepted 27 October 2014

\section{Keywords:}

hip

arthroplasty

length of stay

preoperative predictors

pain

patient expectation

\begin{abstract}
A B S T R A C T
The purpose of this study was to identify preoperative predictors of length of stay after primary total hip arthroplasty in a patient population reflecting current trends toward shorter hospitalization and using readily obtainable factors that do not require scoring systems. A retrospective review of 112 consecutive patients was performed. High preoperative pain level and patient expectation of discharge to extended care facilities (ECFs) were the only significant multivariable predictors of hospitalization extending beyond 2 days $(P=0.001$ and $P<0.001$ respectively). Patient expectation remained significant after adjusting for Medicare's 3-day requirement for discharge to ECFs $(P<0.001)$. The study was adequately powered to analyze the variables in the multivariable logistic regression model, which had a concordance index of 0.857 .
\end{abstract}

(c) 2014 Elsevier Inc. All rights reserved.
Total hip arthroplasty (THA) is one of the most commonly performed and cost-effective orthopedic procedures [1,2]. By 2030, the number of primary THA in the United States is estimated to rise by $174 \%$ to 572,000 procedures [3]. This increased demand will undoubtedly place tremendous strain on existing hospital resources and calls for a greater understanding of the factors associated with hospital length of stay (LOS) to ensure efficient provision of health services.

In recent years, the LOS following THA in the U.S. has decreased substantially, in part due to refined surgical techniques including minimally invasive approaches [4] and implementation of postoperative clinical pathways emphasizing multimodal analgesia and rapid mobilization [5-7]. Medicare's prospective payment system is another incentive for shorter LOS. Medicare provides fixed payments to hospitals based on diagnosis related groups. The longer patients stay, the more money hospitals lose. In response to this financial incentive associated with shorter LOS, there has been an increased utilization of and tendency to discharge to extended care facilities (ECF), such as acute rehabilitation and skilled nursing facilities. Among Medicare beneficiaries, the mean hospital length of stay LOS after THA has decreased from 9.1 days to 3.1 days between 1991 and 2008 with a concomitant four-fold increase in the rate of discharge to ECF [8].

The Conflict of Interest statement associated with this article can be found at http:// dx.doi.org/10.1016/j.arth.2014.10.033.

Reprint requests: Mohamad J. Halawi, MD, Department of Orthopaedic Surgery, Duke University Medical Center Box 3000, Durham, NC 27710.
Previous studies have identified several factors that influence LOS after primary THA, including age [9-12], sex [9,10,12], comorbidities $[12,13]$, body mass index [14], surgical approach [15], use of assistive devices [16], functional status [12], general health perception [10,13], and surgeon experience [10]. However, many of those studies had significantly long mean LOS $[9,10]$, excluded patients with diagnoses other than primary osteoarthritis [16], focused only on select comorbidities [12], or required the use of time-consuming scoring systems $[10,13]$. Furthermore, previous studies often had postoperative rehabilitation protocols geared toward discharge to home [16], which has limited application to current practice where patients with slow postoperative recovery can be discharged to ECF. Most importantly, factors such as preoperative pain level, caregiver assistance, and patient expectation were often not investigated.

Predicting the LOS following THA is important for optimizing hospital resources and helping guide patient expectations. Building on previous studies, the purpose of this investigation was to identify preoperative predictors of LOS after primary THA in a patient population reflecting current trends toward shorter hospitalization and using readily obtainable factors that do not require scoring systems.

\section{Materials and Methods}

This study was approved by the institutional review board at our hospital. The primary outcome of the study was LOS, defined as the number of nights from admission to discharge. Because the standard target for discharge in our practice is postoperative day 2, prolonged 
hospitalization was defined as LOS $>2$. Our institution is a tertiary care teaching hospital performing about 600 THA annually with a large volume of revision and conversion procedures. All patients admitted the three senior authors between January 1, 2012 and December 31, 2012 with CPT code 27130 (THA) as the primary procedure were included in the study. The senior authors are full-time joint arthroplasty surgeons who followed the same postoperative clinical pathway described below. One hundred and twelve patients were identified and their medical records were retrospectively reviewed for factors including age, sex, pain level, body mass index (BMI), number of comorbidities, use of assistive devices, history of prior total hip or knee arthroplasty (TKA), history of prior ECF admission, patient expectation of discharge destination, and caregiver assistance at home. A comorbid condition was defined as any condition requiring active medical treatment. Because a majority of patients carried a diagnosis of arthritis and were receiving medications for joint pain at the time of surgery, arthritis was counted as a comorbid condition only if it involved joints other than the hip that was replaced. We intentionally chose to assess health status based on the number of active conditions in order to eliminate the use of scoring systems, which was one of the goals of this study. Caregiver assistance was classified as either adequate or inadequate depending on whether the caregiver was 1) able and 2) available to provide for patient needs (meals, hygiene, transfers, etc.). Pain level was assessed at the time of preoperative anesthesia evaluation and collected using the numeric rating scale for pain (NRS). NRS is an 11-point scale $(0-10)$ for patient selfreporting of pain with " 0 " indicating no pain and " 10 " indicating the worst imaginable pain. Expectation of discharge destination was collected at the time of initial PT/OT session as described below.

While primary osteoarthritis was the predominant indication for THA, there were no exclusions based on operative diagnosis. Three surgeons performed the operations using either posterior or direct lateral approaches. A variety of cementless implants were used. All patients were admitted from home on the day of surgery. Following THA, patients were managed with a standardized clinical pathway with regards to pain control, mobilization, anticoagulation, and discharge criteria. Spinal anesthesia and single-shot fascia iliaca block were used unless contraindicated. Intravenous patient-controlled analgesia was provided immediately following surgery and transitioned into oral narcotics on the first postoperative day. Unless contraindicated, enoxaparin was used for DVT prophylaxis. Physical and occupational therapy (PT/OT) was initiated on the day of surgery and continued daily until discharge. The primary goals of the first PT/OT session were to assess patients' needs, review acute rehabilitation goals, assist with mobility as tolerated, and initiate preliminary discharge planning. As part of the preliminary discharge planning, the therapists inquired about patients' expectation of discharge destination, which was grouped into either home or ECF (acute rehabilitation and skilled nursing facilities). During the period the hip arthroplasties in this study were performed, patients were provided with informational packets in clinic describing the procedure, benefits, complications, and rehabilitation. They were also given the option of attending a total joint camp, but participation was low and did not exceed 20\%. Preoperatively, patients were informed that their destination of discharge to home versus ECF depended on their progress with PT/OT postoperatively. They were also informed that the target day of discharge to home is the second postoperative day and to ECF the third postoperative day pending the discharge criteria met. Patients were cleared for discharge when they were medically stable, had adequate pain control, were able to void and tolerate oral diet, had no surgical concerns, and were functionally suitable for their discharge destination as determined by PT/OT.

Results for continuous variables were summarized using the mean and standard deviation for normally distributed variables and the 50th (25th, 75th) percentiles or range for non-normally distributed variables. Categorical variables were presented using counts and percentages (rounded to the nearest percent). Continuous variables were tested for normality using the Kolmogorov-Smirnov test. Variables determined to be normally distributed were compared using Student's t-test, while variables non-normally distributed were compared using the Kruskal-Wallis non-parametric test. The relationship between each categorical variable and LOS was assessed using the chi-square test, or Fisher's exact test was utilized in the presence of small cell counts (any expected cell count $<5$ ). All statistical tests were twosided, and a $P$-value $\leq 0.05$ was considered statistically significant. Following univariable analysis, statistically significant factors were subjected to a multivariable stepwise logistic regression analysis to determine the variables associated with LOS. Only variables with $P$-value $\leq 0.05$ remained in the model. The linearity assumption between each continuous variable and the logit of the probability that LOS $>2$ was verified and transformations made if necessary. The concordance index (C-index) was used to determine how well the model discriminated between different responses. The conditional power of the study given the sample size and odds ratio of significant multivariable model covariates was reviewed for statistical adequacy. SAS version 9.2 was used for all analyses.

\section{Results}

One hundred twelve patients were included in this study. There were 58 males and 54 females with a median age of 61.0 years (range 25-85). The median BMI, pain level, and number of comorbidities were 28.6 (range 17.0-47.2), 4.5 (range $0-10$ ), and 2 (range $0-7$ ) respectively. All patients were admitted from home. 41 patients (37\%) used assistive devices prior to surgery. Eight patients (7\%) had prior THA in the contralateral side and 30 patients (27\%) had prior TKA. One hundred four patients (93\%) had adequate caregiver assistance at home. The demographic features of the study cohort are outlined in Table 1.

The mean LOS was 2.5 days (range 2-6). Ninety-two patients (82\%) were discharged to home and 20 (18\%) were discharged to acute rehabilitation centers or skilled nursing facilities. Factors that were not significantly correlated with prolonged LOS were sex $(P=0.062)$, BMI $(P=0.439)$, prior THA in the contralateral limb $(P=0.326)$, and use of assistive devices $(P=0.090)$. Univariable analyses revealed a significant independent association between LOS and number of comorbidities $(P=0.003)$, prior ECF admission $(P=0.037)$, caregiver assistance $(P<0.001)$, and a having a history of TKA $(P=0.017)$; however, these associations did not remain significant after multivariable analysis. Age was a borderline significant multivariable predictor $(P=0.052)$, while pain level $(P=0.001)$ and patient expectation of discharge destination $(P<0.001)$ were the only significant preoperative variables that remained in the final multivariable model. The $\mathrm{C}$-index for the

Table 1

Demographic Features of the Study Group With Univariable Relationship of Each Preoperative Factor With Length Of Stay.

\begin{tabular}{lllr}
\hline Feature & \multicolumn{1}{c}{ LOS $\leq 2$} & \multicolumn{1}{c}{ LOS $>2$} & P Value \\
\hline$N$ & 74 & 38 & \\
Age & $60(54,66)$ & $66.5(57,77)$ & 0.002 \\
$\quad$ Male & $43(58 \%)$ & $15(40 \%)$ & 0.062 \\
$\quad$ Female & $31(42 \%)$ & $23(60 \%)$ & \\
BMI & $28.6(25.2,32.2)$ & $28.6(19.1,36.1)$ & 0.439 \\
Pain level & $4(1,5)$ & $6.5(5,8)$ & $<0.001$ \\
Number of comorbidities & $2(1,3)$ & $3(2,4)$ & 0.003 \\
Use of assistive device & $23(31 \%)$ & $18(47 \%)$ & 0.090 \\
History of ECF admission & $0(0 \%)$ & $3(8 \%)$ & 0.037 \\
History of THA & $22(30 \%)$ & $8(21 \%)$ & 0.326 \\
History of TKA & $2(3 \%)$ & $6(16 \%)$ & 0.018 \\
Assistance at home & & & $<0.001$ \\
$\quad$ Adequate & $74(100 \%)$ & $30(79 \%)$ & \\
$\quad$ Inadequate & $0(0 \%)$ & $8(21 \%)$ & \\
Expectation of discharge destination & & & \\
$\quad$ Home & $73(99 \%)$ & $19(50 \%)$ & \\
$\quad$ ECF & $1(1 \%)$ & $19(50 \%)$ & \\
\hline
\end{tabular}

Data are presented as $N$ (\%) or 50th (25th, 75 th) percentiles.

$\mathrm{ECF}=$ extended care facility. 
multivariable logistic regression model was 0.857 . The multivariable model had more than $80 \%$ power to detect a significant difference in pain level and patient expectation. Preoperative pain level $>3$ was a significant cut point associated with increased LOS $(P<0.05$; Fig. 1$)$. Tables 1 and 2 outline both the univariable and multivariable relationships of each preoperative factor with LOS. In a subset analysis, patient expectation of discharge to ECF remained predictive of prolonged hospitalization when target LOS was adjusted to 3 days $(P<0.001)$, thereby controlling for any potential confounding factors, such as Medicare's 3-day rule to quality for discharge to ECF.

Twelve patients (11\%) developed minor postoperative complications during hospitalization, mostly narcotic-related adverse drug events. There was a significant association between LOS and the development of a complication $(P<0.001)$. Four patients $(4 \%)$ had blood transfusion; however, there was no significant relationship between LOS and blood transfusion $(P=0.112)$. Patients with longer LOS had significantly higher rate of discharge to $\mathrm{ECF}(P<0.001)$.

\section{Discussion}

Understanding the preoperative factors associated with LOS following THA may permit increased efficiency of patient flow, reduction of healthcare costs, better management of patient expectations, and increased patient satisfaction. Although a few studies have been published, the relatively long LOS and broad exclusion criteria in those studies limit their utility to current practice. Most importantly, factors such as preoperative pain level and patient expectation were often not investigated.

In this study, we identified a significant factor not previously reported. Contrary to Dall et al [10], we found a significant association between LOS and preoperative pain level. Dall et al studied pain level as measured by the SF-36 pain score; however, their mean LOS was 8.1 days and no physical therapy was provided on weekends, which make direct comparison difficult. Their study population was also exclusively Caucasian and the rehabilitation milestones were geared toward discharge to home. The relationship between higher pain levels and prolonged hospitalization should come as no surprise with mounting evidence showing shorter LOS after implementation of multimodal analgesia protocols [6,17]. In our patient population, there was a trend toward chronic narcotics use in patients who reported higher preoperative pain levels. Most importantly, among the patients who had poor postoperative pain control or narcotic-related side-effects (ileus, nausea, delirium, etc.) that prolonged their hospitalization, all reported high preoperative pain levels. The relationship between LOS and inadequate postoperative pain control or development of an adverse event related to narcotics was significant $(P<0.001)$.

The effect of patient expectation of discharge destination on LOS has also never been previously reported. This variable remained a significant predictor of LOS even beyond the third postoperative day, thereby eliminating any potential confounding effect of Medicare's 3-day

Estimated Spline Transformation and 95\% C.I.

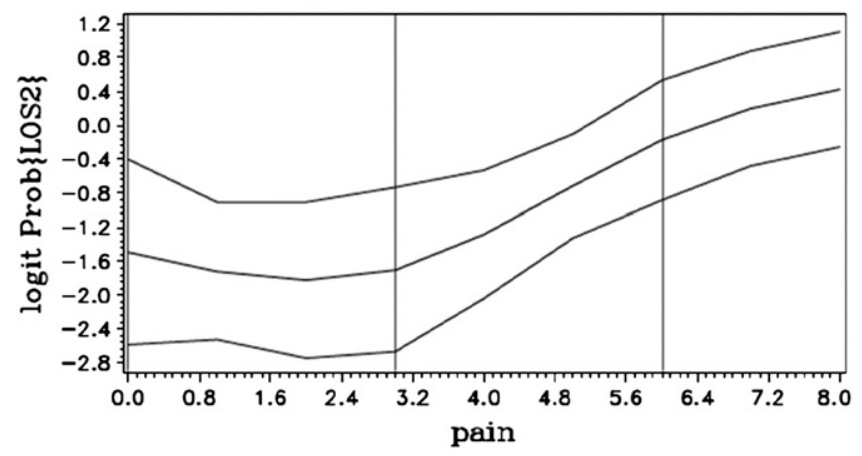

Fig. 1. Pain level $>3$ was a significant cut-off point for increased likelihood of longer length of stay $(P>0.05)$
Table 2

Multivariable Logistic Regression Model for Predictors of LOS >2 Days.

\begin{tabular}{lrc}
\hline Factor & $P$ Value & Odds Ratio (95\% Confidence Interval) \\
\hline Pain level & 0.001 & $1.53(1.19-1.96)$ \\
Expectation of discharge to ECF & $<0.001$ & $77.70(9.22-655.14)$ \\
\hline
\end{tabular}

$\mathrm{ECF}=$ extended care facility.

inpatient stay requirement for discharge to post-acute care facilities. This finding is especially interesting. It demonstrates that a patient's notion of care needs is more powerful than caregiver support, reliance on mobility aids, or comorbidities (all of which were not significant in our study). Patient expectation is a subjective measure of one's mindset and can be influenced by complex variables, such as anxiety, selfconfidence, and knowledge. Meeting patient expectation has been shown to strongly correlate with patient satisfaction $[18,19]$.

There are several weaknesses in this study. First, it was a retrospective chart review from a single tertiary-care teaching institution. Compared to a prospective design, however, this retrospective analysis may have offered the advantage of eliminating observer bias as the data were collected without the intent of the study at that time. An outcome such as LOS could have been easily influenced by the study personnel if it were prospectively assessed. Second, our patient sample was small compared to previously published studies that were based on large-scale database searches, but we had sufficient power for the statistically significant factors in the multivariable logistic regression model. In addition, the C-index, which is a measure of how well the multivariable logistic regression model discriminated between different responses, was quite good. Third, our list of preoperative variables was not all-inclusive and factors such as patients' home environment (stairs, number of residents, etc.) were not assessed. Those factors were not consistently available in the charts and hence were not analyzed. Fourth, the use of number of active comorbidities as opposed to a more well recognized and validated comorbidity scoring system, such as the Charlson comorbidity index (CCI) may represent a weakness. However, there is currently no gold-standard instrument for evaluating comorbidities and the $\mathrm{CCI}$ is not all-inclusive [20]. Specifically, while the $\mathrm{CCI}$ is an excellent prognostic tool in life-threatening conditions as it was initially designed [21,22], it may not be sensitive enough for lowmortality elective procedures, such as THA [23] and it does not account for conditions that may impact the postoperative course, such as depression, anxiety, arthritis, fibromyalgia, and back pain. In addition, our results were consistent with earlier studies that found no significant link between comorbidities and LOS after THA. These studies used different measures of patient comorbidity including the $\mathrm{CCI}$ and counting the number of comorbidities [24].

We believe that this study has several strengths. The target discharge date on the second postoperative day reflects current fast-track trends [25]. We analyzed a consecutive group of patients who underwent primary unilateral THA without exclusion criteria. Patients underwent a standardized postoperative clinical pathway with clear discharge criteria. All the preoperative factors are easily obtainable with no need for scoring systems. By reviewing individual patient charts, we had the advantage of assessing factors that are sometimes not routinely collected in large-scale databases, such as caregiver assistance, patient expectations, and pain level.

Our study has demonstrated for the first time that high preoperative pain level and patient expectation of discharge to ECF are significant predictors of increased hospitalization based on a target LOS of 2 days. Patient expectation of discharge to ECF remained a significant predictor of increased LOS even after controlling for Medicare's 3-day rule to quality for discharge to ECF. We have also confirmed earlier findings, particularly the association between LOS and age [9-12] and absence of association between LOS and BMI, sex, and comorbidities $[10,13,16]$. Pain and patient expectations represent compelling targets for intervention to reduce LOS. 


\section{References}

1. Jenkins PJ, Clement ND, Hamilton DF, et al. Predicting the cost-effectiveness of total hip and knee replacement: a health economic analysis. Bone Joint J 2013;95-B(1):115.

2. Lavernia CJ, Alcerro JC. Quality of life and cost-effectiveness 1 year after total hip arthroplasty. J Arthroplast 2011;26(5):705.

3. Kurtz S, Ong K, Lau E, et al. Projections of primary and revision hip and knee arthroplasty in the United States from 2005 to 2030. J Bone Joint Surg Am 2007;89(4):780.

4. Rottger J, Scheller-Kreinsen D, Busse R. Patient-level hospital costs and length of stay after conventional versus minimally invasive total hip replacement: a propensitymatched analysis. Value Health 2012;15(8):999.

5. Malviya A, Martin K, Harper I, et al. Enhanced recovery program for hip and knee replacement reduces death rate. Acta Orthop 2011;82(5):577.

6. Duellman TJ, Gaffigan C, Milbrandt JC, et al. Multi-modal, pre-emptive analgesia decreases the length of hospital stay following total joint arthroplasty. Orthopedics 2009;32(3):167.

7. Tayrose G, Newman D, Slover J, et al. Rapid mobilization decreases length-of-stay in joint replacement patients. Bull Hosp Joint Dis 2013;71(3):222.

8. Cram P, Lu X, Kaboli PJ, et al. Clinical characteristics and outcomes of Medicare patients undergoing total hip arthroplasty, 1991-2008. JAMA 2011;305(15):1560.

9. Abbas K, Umer M, Qadir I, et al. Predictors of length of hospital stay after total hip replacement. J Orthop Surg 2011;19(3):284.

10. Dall GF, Ohly NE, Ballantyne JA, et al. The influence of pre-operative factors on the length of in-patient stay following primary total hip replacement for osteoarthritis: a multivariate analysis of 2302 patients. J Bone Joint Surg Br 2009;91(4):434.

11. Forrest G, Fuchs M, Gutierrez A, et al. Factors affecting length of stay and need for rehabilitation after hip and knee arthroplasty. J Arthroplast 1998;13(2):186.

12. O'Malley NT, Fleming FJ, Gunzler DD, et al. Factors independently associated with complications and length of stay after hip arthroplasty: analysis of the National Surgical Quality Improvement Program. J Arthroplast 2012;27(10):1832.

13. Styron JF, Koroukian SM, Klika AK, et al. Patient vs provider characteristics impacting hospital lengths of stay after total knee or hip arthroplasty. J Arthroplast 2011;26(8): 1418 [e1411-1412]
14. Maradit Kremers H, Visscher SL, Kremers WK, et al. Obesity increases length of stay and direct medical costs in total hip arthroplasty. Clin Orthop Relat Res 2014; 472(4):1232.

15. Martin CT, Pugely AJ, Gao Y, et al. A comparison of hospital length of stay and shortterm morbidity between the anterior and the posterior approaches to total hip arthroplasty. J Arthroplast 2013;28(5):849.

16. van Aalst MJ, Oosterhof J, Nijhuis-van der Sanden MW, et al. Can the length of hospital stay after total hip arthroplasty be predicted by preoperative physical function characteristics? Am J Phys Med Rehabil 2014;93:486.

17. Peters CL, Shirley B, Erickson J. The effect of a new multimodal perioperative anesthetic regimen on postoperative pain, side effects, rehabilitation, and length of hospital stay after total joint arthroplasty. J Arthroplast 2006:21(6 Suppl 2):132.

18. Mancuso CA, Salvati EA, Johanson NA, et al. Patients' expectations and satisfaction with total hip arthroplasty. J Arthroplast 1997;12(4):387.

19. Mahomed NN, Liang MH, Cook EF, et al. The importance of patient expectations in predicting functional outcomes after total joint arthroplasty. J Rheumatol 2002; 29(6):1273.

20. Bjorgul K, Novicoff WM, Saleh KJ. Evaluating comorbidities in total hip and knee arthroplasty: available instruments. J Orthop Traumatol 2010;11(4):203.

21. Charlson ME, Pompei P, Ales KL, et al. A new method of classifying prognostic comorbidity in longitudinal studies: development and validation. J Chronic Dis 1987;40(5):373.

22. Kastner C, Armitage J, Kimble A, et al. The Charlson comorbidity score: a superior comorbidity assessment tool for the prostate cancer multidisciplinary meeting. Prostate Cancer Prostatic Dis 2006;9(3):270.

23. Harse JD, Holman CD. Charlson's Index was a poor predictor of quality of life outcomes in a study of patients following joint replacement surgery. J Clin Epidemiol 2005;58(11):1142

24. Olthof M, Stevens M, Bulstra SK, et al. The association between comorbidity and length of hospital stay and costs in total hip arthroplasty patients: a systematic review. J Arthroplast 2014;29(5):1009.

25. Gulotta LV, Padgett DE, Sculco TP, et al. One hospital's experience with a 2-day length of stay protocol for total hip replacement. HSS J 2011;7(3):223. 